\title{
Bochner definition of Stepanov-like almost automorphic functions on time scales and an application to cellular neural networks with delays
}

\section{Li-Li Zhang $^{1 *}$ (D) and Xu-Dong Yang ${ }^{1}$}

"Correspondence:

zllyou0@gmail.com

${ }^{1}$ Department of Mathematics and

Physics, Shijiazhuang Tiedao

University, Shijiazhuang, Hebei

050043, P.R. China

\section{글 Springer}

\begin{abstract}
The definition of Stepanov-like almost automorphic functions on time scales had been proposed in the literature, but at least one result was incorrect, which involved Bochner transform. In our work, we give the Bochner definition of Stepanov-like almost automorphic functions on time scales, and prove that a function is Stepanov-like almost automorphic if and only if it satisfies Bochner definition of Stepanov-like almost automorphic function on time scales. The Bochner definition of Stepanov-like almost automorphic functions on time scales corrects the faulty result, and perfects the definition of Stepanov-like almost automorphic functions. As applications, we discuss the almost automorphy of a certain dynamic equation and some cellular neural networks with delays on time scales.
\end{abstract}

MSC: $34 \mathrm{~K} 14 ; 34 \mathrm{~N} 05$

Keywords: Time scale; Bochner-like transform; Stepanov-like almost automorphic function; Dynamic equation; Cellular neural networks

\section{Introduction}

In 1988, S. Hilger first introduced the theory of time scales in his PhD thesis (see [1]), which unifies continuous and discrete situations. Thus the field of differential equations on time scales can extend the classical differential equations and difference equations on $\mathbb{R}$. Moreover, this theory provides important tools in the areas of economics, populations models, quantum physics, and some others. Therefore, many researchers are interested in this theory (see [2-5]). Dynamic systems and cellular neural networks are two important models in applications. In recent years, the investigations of the above two systems on time scales have attracted many mathematicians' attention.

The theory of almost periodicity on time scales dates back to 2011. The authors of [6,7] first extended the classical almost periodic functions on $\mathbb{R}$ to time scales, and also applied them to investigate the almost periodicity of some high-order Hopfield neural networks on time scales. Thereafter, the theory of almost periodicity on time scales has attracted

(c) The Author(s) 2021. This article is licensed under a Creative Commons Attribution 4.0 International License, which permits use, sharing, adaptation, distribution and reproduction in any medium or format, as long as you give appropriate credit to the original author(s) and the source, provide a link to the Creative Commons licence, and indicate if changes were made. The images or other third party material in this article are included in the article's Creative Commons licence, unless indicated otherwise in a credit line to the material. If material is not included in the article's Creative Commons licence and your intended use is not permitted by statutory regulation or exceeds the permitted use, you will need to obtain permission directly from the copyright holder. To view a copy of this licence, visit http://creativecommons.org/licenses/by/4.0/ 
attention of many scientists. Lizama, Mesquita, and Ponce [8] gave a related concept of almost periodicity on time scales, and also constructed one almost periodic function on $\mathbb{R}$ as well as on time scales. These results enriched the theory of almost periodicity. As the development of the theory of almost periodicity on $\mathbb{R}$, generalizations of the definition of an almost periodic function on time scales are also interesting topics. Li and Wang defined pseudo almost periodicity in [9], and then, Li and Zhao investigated weighted pseudo periodic functions [10]. Meanwhile, Lizama and Mesquita [11] defined almost automorphy on time scales. Furthermore, the Stepanov-like definitions were also suggested. Tang and Li $[12,13]$ gave the concept of Stepanov-like almost periodic functions and Stepanovlike pseudo almost periodic functions on time scales, and Dhama and Abbas [14] showed weighted Stepanov-like pseudo almost automorphic functions on time scales. In the definition of Stepanov-like functions on $\mathbb{R}$, Bochner transform is an important tool, but it is not available in the investigation on time scales. To solve this problem, Tang and $\mathrm{Li}$ [12] proposed the definition of Bochner-like transform on time scales. At least one result in [14] involving the Bochner definition was not true, and so it is important to give the Bochner definition of Stepanov-like almost automorphic functions on time scales.

The first contribution of our work is an introduction of the Bochner definition of Stepanov-like almost automorphic functions on time scales by applying a Bochner-like transform, which is equivalent to the earlier definition of Stepanov-like almost automorphic functions on time scales provided in [14]. Our definition corrects the result in [14]. Secondly, as applications, we present a study of the almost automorphy for the following dynamic equation:

$$
u^{\Delta}(s)=\mathcal{A}(s) u(s)+\varphi(s), \quad s \in \mathbb{T},
$$

with $\mathcal{A}(s)$ being almost automorphic, and $\varphi(s)$ Stepanov-like almost automorphic on $\mathbb{T}$. The almost automorphy [11] and pseudo almost periodicity [13] of equation (1.1) have been studied, with $\varphi(s)$ being almost automorphic and pseudo almost periodic, respectively. Our results extend earlier investigations. Finally, we show a result about the almost automorphy for the following system:

$$
u_{l}^{\Delta}(s)=-a_{l}(s) u_{l}(s)+\sum_{m=1}^{n} b_{l m}(s) F_{m}\left(u_{m}(s)\right)+\sum_{m=1}^{n} c_{l m}(s) F_{m}\left(u_{m}\left(s-\gamma_{l m}\right)\right)+I_{l}(s)
$$

for $s \in \mathbb{T}, l=1,2, \ldots, n$. This system is called a cellular neural network, where $u_{l}(s)$ denotes the activation of the $l$ th neuron at time $s, a_{l}(s)$ corresponds to the rate cell $l$ resets its potential to the resting state when isolated from the other cells and inputs at time $s, \gamma_{l m}$ denotes the transmission delay, $I_{l}(s)$ and $F_{m}$ correspond to the external input and the activation function, respectively, and $b_{l m}(s)$ and $c_{l m}(s)$ are the connection weights at time $s$.

Our work is divided into four parts. In the second section, we present preliminaries, including the definitions and properties of Stepanov-like almost automorphic functions on time scales. The third section is devoted to establishing the result about the almost automorphy for some linear dynamic equation on time scales. At last, we show the existence of an almost automorphic solution for some cellular neural networks with delays, and present an example. 


\section{Definitions and properties}

In the sequel, we set

$$
\begin{aligned}
& \mathbb{Z}=\{s: s \text { is a integer }\}, \quad \mathbb{R}=\{s: s \text { is a real number }\}, \\
& \mathbb{C}=\{s: s \text { is a complex number }\}, \quad \mathbb{R}^{+}=\{s: s \text { is a real number, and } s \geq 0\} .
\end{aligned}
$$

The Euclidean space $\mathbb{R}^{n}$ is equipped with Euclidean norm $|\cdot|$. Let $X$ be a Banach space with the norm $\|\cdot\|$.

We will show some definitions about time scales, which are taken from [2, 4]. If $\mathbb{T}$ is a nonempty closed subset of $\mathbb{R}$, we say it is a time scale. Let $s \in \mathbb{T}$, the forward jump operator $\sigma: \mathbb{T} \rightarrow \mathbb{T}$ and backward jump operator $\rho: \mathbb{T} \rightarrow \mathbb{T}$ are defined by

$$
\sigma(s)=\inf \{\tau \in \mathbb{T}, \tau>s\}, \quad \rho(s)=\sup \{\tau \in \mathbb{T}, \tau<s\} .
$$

If $\rho(s)=s$, we call $s$ left-dense, and otherwise, we call it left-scattered. Similarly, if $\sigma(s)=s$, $s \in \mathbb{T}$ is called right-dense. Otherwise, $s \in \mathbb{T}$ is said to be right-scattered. Now we show the definition of the graininess $\mu: \mathbb{T} \rightarrow \mathbb{R}^{+}$with $\mu(s)=\sigma(s)-s$. If $\alpha$ is a left-scattered maximum of $\mathbb{T}$, we denote $\mathbb{T}^{k}=\mathbb{T} \backslash\{\alpha\}$; otherwise $\mathbb{T}^{k}=\mathbb{T}$.

Now we present the definition of a continuous function and delta derivative on time scales.

Definition 2.1 ([11]) (i) $\varphi: \mathbb{T} \rightarrow X$ is said to be rd-continuous if it is continuous at each right-dense point and the left-hand side limits exist at left-dense points.

(ii) $\varphi: \mathbb{T} \rightarrow X$ is called continuous on $\mathbb{T}$ if it is continuous at both right- and left-dense points.

Set

$$
\begin{aligned}
& C_{r d}(\mathbb{T}, X)=\{\varphi: \mathbb{T} \rightarrow X: \varphi \text { is right-dense continuous }\}, \\
& L_{\text {loc }}^{p}(\mathbb{T}, X)=\left\{\varphi: \mathbb{T} \rightarrow X: \varphi \text { is locally } L^{p} \Delta \text {-integrable }\right\} .
\end{aligned}
$$

Let $a_{1}, a_{2} \in \mathbb{T}$ and $a_{1} \leq a_{2},\left[a_{1}, a_{2}\right]_{\mathbb{T}}=\left\{s \in \mathbb{T}: a_{1} \leq s \leq a_{2}\right\}$, and $\left[a_{1}, a_{2}\right]$ be the usual interval on $\mathbb{R}$.

Definition 2.2 ([2]) Let $\varphi: \mathbb{T} \rightarrow X$ and $s \in \mathbb{T}^{k}$. If $\varphi^{\Delta}(s) \in X$ is such that for any positive constant $\varepsilon$ we can find a neighborhood $U_{s}$ of $s$, satisfying

$$
\left\|\varphi(\sigma(s))-\varphi(\tau)-\varphi^{\Delta}(s)(\sigma(s)-\tau)\right\|<\varepsilon|\sigma(s)-\tau|, \quad \tau \in U_{s},
$$

we call $\varphi^{\Delta}(s)$ the delta derivative of $\varphi(s)$.

The definition of integration on time scales is summarized in [12], and the authors of from [3] showed that the integral $\int_{\left[a_{1}, a_{2}\right]_{\mathbb{T}}} \varphi(s) \Delta s$ also satisfies the Lebesgue integration theory on $\mathbb{T}$.

Definition $2.3([6,7,11])$ Time scale $\mathbb{T}$ is said to be invariant under translations if the set $\Pi$ is a nonempty set, where

$$
\Pi=\{\tau \in \mathbb{R}: s \pm \tau \in \mathbb{T}, \forall s \in \mathbb{T}\}
$$


In the following, we always suppose $\mathbb{T}$ is invariant under translation. In [12], the authors gave its properties. Set

$$
L:=\inf \{|\tau|: \tau \in \Pi, \tau \neq 0\} .
$$

Lemma 2.1 ([12]) $L>0$ if and only if $\mathbb{T} \neq \mathbb{R}$, and $L=0$ if and only if $\mathbb{T}=\mathbb{R}$. Furthermore, the following result is true:

$$
\Pi= \begin{cases}L \mathbb{Z} & \text { for } \mathbb{T} \neq \mathbb{R} \\ \mathbb{R} & \text { for } \mathbb{T}=\mathbb{R} .\end{cases}
$$

Now we are in the position to give the definition and propositions for Stepanov-like almost automorphic functions on $\mathbb{T}$.

Definition 2.4 ([11]) Let $\varphi \in C_{r d}(\mathbb{T}, X)$. If for each $\left\{\alpha_{n}^{\prime}\right\}$ in $\Pi$ there are a subsequence $\left\{\alpha_{n}\right\} \subset\left\{\alpha_{n}^{\prime}\right\}$ and a function $\tilde{\varphi}$ satisfying

$$
\lim _{n \rightarrow \infty} \varphi\left(s+\alpha_{n}\right)=\tilde{\varphi}(s) \quad \text { and } \quad \lim _{n \rightarrow \infty} \tilde{\varphi}\left(s-\alpha_{n}\right)=\varphi(s)
$$

pointwise on $\mathbb{T}$, we call $\varphi$ an almost automorphic function on $\mathbb{T}$, and denote their space by $A A(\mathbb{T}, X)$.

Define

$$
\mathcal{L}:= \begin{cases}1 & \text { for } \mathbb{T}=\mathbb{R} \\ L & \text { for } \mathbb{T} \neq \mathbb{R}\end{cases}
$$

with $L$ defined in (2.1). In the following, we work under the assumption that $p \geq 1$. Define the norm $\|\cdot\|_{S^{p}}$ as

$$
\|\varphi\|_{S^{p}}:=\sup _{s \in \mathbb{T}}\left(\frac{1}{\mathcal{L}} \int_{[s, s+\mathcal{L}]_{\mathbb{T}}}\|\varphi(r)\|^{p} \Delta r\right)^{\frac{1}{p}} \text { for } \varphi \in L_{\mathrm{loc}}^{p}(\mathbb{T}, X) .
$$

If $\|\varphi\|_{S^{p}}<\infty$, we call function $\varphi S^{p}$-bounded, and denote their space by $B S^{p}(\mathbb{T}, X)$.

Remark 2.1 In [12], the authors have shown some results about the space $B S^{p}(\mathbb{T}, X)$. That is,

(i) $\left(B S^{p}(\mathbb{T}, X),\|\cdot\|_{S^{p}}\right)$ is a Banach space.

(ii) $B S^{p}(\mathbb{T}, X)$ is translation invariant.

(iii) $B S^{p}(\mathbb{T}, X) \subset B S^{q}(\mathbb{T}, X)$ for $1 \leq q \leq p<\infty$.

Definition 2.5 ([14]) A function $\varphi \in B S^{p}(\mathbb{T}, X)$ is said to be Stepanov-like almost automorphic if for every sequence $\left\{\alpha_{n}^{\prime}\right\} \in \Pi$, we can extract a subsequence $\left\{\alpha_{n}\right\}$ and find a function $\tilde{\varphi} \in L_{l o c}^{p}(\mathbb{T}, X)$ such that

$$
\left\|\varphi\left(s+\alpha_{n}\right)-\tilde{\varphi}(s)\right\|_{S^{p}} \rightarrow 0, \quad\left\|\tilde{\varphi}\left(s-\alpha_{n}\right)-\varphi(s)\right\|_{S^{p}} \rightarrow 0 \quad \text { as } n \rightarrow \infty .
$$


In [14], the authors claimed the following conclusion:

(A) $\varphi \in S^{p} A A(\mathbb{T}, X)$ if and only if $\bar{\varphi} \in A A\left(\mathbb{T}, L^{p}\left([0, \mathcal{L}]_{\Pi}, X\right)\right)$, where $\bar{\varphi}(s, t)=\varphi(s+t)$, with $s \in \mathbb{T}, t \in[0, \mathcal{L}]_{\Pi}$.

But the result is not true, and the following example shows it.

Example 2.1 Set $X=\mathbb{R}$, and

$$
\mathbb{T}=\bigcup_{i=0}^{\infty}[i(a+b), i(a+b)+a],
$$

with $a, b>0$. It is clear that $\mathcal{L}=a+b, \Pi=(a+b) \mathbb{Z}$, and $[0, \mathcal{L}]_{\Pi}=\{0, a+b\}$. On time scale $\Pi, \mu(0)=\mu(a+b)=a+b$. Set

$$
\varphi(s)= \begin{cases}s^{2}, & s \in(a+b) \mathbb{Z}, \\ \sin \frac{1}{2+\cos s+\cos \pi s}, & s \in \mathbb{T} \backslash(a+b) \mathbb{Z} .\end{cases}
$$

We can check that $\varphi \in S^{p} A A(\mathbb{T}, \mathbb{R})$. But

$$
\begin{aligned}
\|\bar{\varphi}\|_{\infty} & =\sup _{s \in \mathbb{T}}\|\bar{\varphi}(s, t)\|_{L^{p}\left([0, \mathcal{L}]_{\Pi} ; \mathbb{R}\right)}=\sup _{s \in \mathbb{T}}\left(\int_{[0, \mathcal{L}]_{\Pi}}|\varphi(s+t)|^{p} \Delta t\right)^{\frac{1}{p}} \\
& =\sup _{s \in \mathbb{T}}\left(\mu(0)|\varphi(s)|^{p}+\mu(a+b)|\varphi(a+b+s)|^{p}\right)^{\frac{1}{p}} \geq \sup _{s \in \mathbb{T}}|\varphi(s)|=\infty .
\end{aligned}
$$

That is, $\bar{\varphi} \notin A A\left(\mathbb{T}, L^{p}\left([0, \mathcal{L}]_{\Pi}, \mathbb{R}\right)\right)$, and the conclusion $(\mathrm{A})$ is not true.

It is important to introduce the Bochner definition of Stepanov-like almost automorphic functions. In the following, we will give the Bochner definition of Stepanov-like almost automorphic functions on time scales by applying a Bochner-like transform which is introduced in [12].

If $\mathbb{T} \neq \mathbb{R}$, let $\omega$ be a left-scattered point in $\mathbb{T}$. For any fixed $s \in \mathbb{T}$, it is not difficult to verify that there exists a unique integer $n_{s}$ such that $s-n_{s} \mathcal{L} \in[\omega, \omega+\mathcal{L})_{\mathbb{T}}$.

Definition 2.6 ([12]) Let $\varphi \in B S^{p}(\mathbb{T}, X)$. Define $\varphi^{c}: \mathbb{T} \times \mathbb{T} \rightarrow X$ by $\varphi^{c}(s, t)=\varphi\left(\mathcal{N}_{s} \mathcal{L}+t\right)$ for $s, t \in \mathbb{T}$, where

$$
\mathcal{N}_{s}= \begin{cases}s, & \mathbb{T}=\mathbb{R} \\ n_{s}, & \mathbb{T} \neq \mathbb{R} .\end{cases}
$$

We call $\varphi^{c}$ the Bochner-like transform of $\varphi$.

By using Bochner-like transform, we can present the following Bochner definition:

Definition 2.7 Let $\varphi \in B S^{p}(\mathbb{T}, X)$. If the Bochner-like transform $\varphi^{c} \in A A\left(\mathbb{T}, B S^{p}(\mathbb{T}, X)\right.$ ), we say that $\varphi$ is Stepanov-like almost automorphic on $\mathbb{T}$ based on Bocher definition. Denote their set by $S^{p} A A(\mathbb{T}, X)$.

Lemma 2.2 $\mathcal{N}_{s+\alpha} \mathcal{L}=\mathcal{N}_{s} \mathcal{L}+\alpha$ for $s \in \mathbb{T}$ and $\alpha \in \Pi$. 
Proof If $\mathbb{T}=\mathbb{R}$, we can easily get $\mathcal{N}_{s+\alpha} \mathcal{L}=s+\alpha=\mathcal{N}_{s} \mathcal{L}+\alpha$, and the conclusion is true. If $\mathbb{T} \neq \mathbb{R}$, we know $\mathcal{L}=L$. From Lemma 2.1, there is $\alpha^{\prime} \in \mathbb{Z}$ such that $\alpha=\alpha^{\prime} L$. Since $s-n_{s} L \in$ $[\omega, \omega+L)_{\mathbb{T}}$, we have

$$
s+\alpha-\alpha^{\prime} L-n_{s} L=s+\alpha-\left(n_{s}+\alpha^{\prime}\right) L=s-n_{s} L \in[\omega, \omega+L)_{\mathbb{T}} .
$$

Since $n_{s+\alpha}$ is unique, we have $n_{s+\alpha}=n_{s}+\alpha^{\prime}$, thus $n_{s+\alpha} L=n_{s} L+\alpha$, and this completes the proof.

Theorem 2.1 For $\varphi \in B S^{p}(\mathbb{T}, X)$, the following statements are equivalent:

(i) $\varphi \in S^{p} A A(\mathbb{T}, X)$.

(ii) For each $\left\{\alpha_{n}^{\prime}\right\}$ in $\Pi$, we can find $\left\{\alpha_{n}\right\} \subset\left\{\alpha_{n}^{\prime}\right\}$ and $\tilde{\varphi} \in B S^{p}(\mathbb{T}, X)$ such that

$$
\begin{aligned}
& \lim _{n \rightarrow \infty}\left(\frac{1}{\mathcal{L}} \int_{[s, s+\mathcal{L}]_{\mathbb{T}}}\left\|\varphi\left(r+\alpha_{n}\right)-\tilde{\varphi}(r)\right\|^{p} \Delta r\right)^{\frac{1}{p}}=0 \quad \text { and } \\
& \lim _{n \rightarrow \infty}\left(\frac{1}{\mathcal{L}} \int_{[s, s+\mathcal{L}]_{\mathbb{T}}}\left\|\tilde{\varphi}\left(r-\alpha_{n}\right)-\varphi(r)\right\|^{p} \Delta r\right)^{\frac{1}{p}}=0
\end{aligned}
$$

pointwise on $\mathbb{T}$.

Proof For $\mathbb{T} \neq \mathbb{R}$, let $\varphi, \tilde{\varphi} \in B S^{p}(\mathbb{T}, X)$, and consider a sequence $\left\{\alpha_{n}\right\} \subset \Pi$. From Lemma 2.2, for each $s \in \mathbb{T}$, we have

$$
\begin{aligned}
& \left(\frac{1}{\mathcal{L}} \int_{[s, s+\mathcal{L}]_{\mathbb{T}}}\left\|\varphi\left(r+\alpha_{n}\right)-\tilde{\varphi}(r)\right\|^{p} \Delta r\right)^{\frac{1}{p}} \\
& \quad=\sup _{\tau \in \mathbb{T}}\left(\frac{1}{\mathcal{L}} \int_{\left[\tau+\mathcal{N}_{s} \mathcal{L}, \tau+\mathcal{N}_{s} \mathcal{L}+\mathcal{L}\right]_{\mathbb{T}}}\left\|\varphi\left(r+\alpha_{n}\right)-\tilde{\varphi}(r)\right\|^{p} \Delta r\right)^{\frac{1}{p}} \\
& =\sup _{\tau \in \mathbb{T}}\left(\frac{1}{\mathcal{L}} \int_{[\tau, \tau+\mathcal{L}]_{\mathbb{T}}}\left\|\varphi\left(\mathcal{N}_{s} \mathcal{L}+\alpha_{n}+r\right)-\tilde{\varphi}\left(\mathcal{N}_{s} \mathcal{L}+r\right)\right\|^{p} \Delta r\right)^{\frac{1}{p}} \\
& =\sup _{\tau \in \mathbb{T}}\left(\frac{1}{\mathcal{L}} \int_{[\tau, \tau+\mathcal{L}]_{\mathbb{T}}}\left\|\varphi\left(\mathcal{N}_{s+\alpha_{n}} \mathcal{L}+r\right)-\tilde{\varphi}\left(\mathcal{N}_{s} \mathcal{L}+r\right)\right\|^{p} \Delta r\right)^{\frac{1}{p}} \\
& =\left\|\varphi^{c}\left(s+\alpha_{n}, \cdot\right)-\tilde{\varphi}^{c}(s, \cdot)\right\|_{S^{p}} .
\end{aligned}
$$

Thus the two statements are equivalent for $\mathbb{T} \neq \mathbb{R}$. For $\mathbb{T}=\mathbb{R}$, it is clear that $\varphi^{c}(s, t)=\varphi(s+t)$ for $s, t \in \mathbb{R}$. From the above argument, we know the two statements are equivalent, and this completes the proof.

Remark 2.2 (i) Clearly, we can get that $A A(\mathbb{T}, X) \subset S^{p} A A(\mathbb{T}, X)$.

(ii) By the above Theorem 2.1, we can easily get the following result: 
$\varphi \in S^{p} A A(\mathbb{T}, X)$ if and only if for each sequence $\left\{\alpha_{n}^{\prime}\right\}$ in $\Pi$, there exist $\left\{\alpha_{n}\right\} \subset\left\{\alpha_{n}^{\prime}\right\}$ and $\tilde{\varphi} \in B S^{p}(\mathbb{T}, X)$ such that

$$
\begin{aligned}
& \left(\frac{1}{\mathcal{L}} \int_{[s, s+\mathcal{L}]_{\mathbb{T}}}\left\|\varphi\left(r+\alpha_{n}\right)-\tilde{\varphi}(r)\right\|^{p} \Delta r\right)^{\frac{1}{p}} \rightarrow 0 \text { and } \\
& \left(\frac{1}{\mathcal{L}} \int_{[s, s+\mathcal{L}]_{\mathbb{T}}}\left\|\tilde{\varphi}\left(r-\alpha_{n}\right)-\varphi(r)\right\|^{p} \Delta r\right)^{\frac{1}{p}} \rightarrow 0
\end{aligned}
$$

as $n \rightarrow \infty$ pointwise on $\mathbb{T}$. Thus the Bochner definition of Stepanov-like almost automorphic function is equivalent to Definition 2.12 in [14].

(iii) If the convergent "pointwise" in (ii) is changed to "uniformly", we know it is Stepanov-like almost periodic on $\mathbb{T}$. Thus $S^{p} A P(\mathbb{T}, X) \subset S^{p} A A(\mathbb{T}, X)$.

Remark 2.3 $\varphi \in S^{p} A A(\mathbb{Z}, X)$ if and only if $\varphi \in A A(\mathbb{Z}, X)$. Indeed, letting $\mathbb{T}=\mathbb{Z}$, we can easily get that $\Pi=\mathbb{Z}, L=1$, and $\mu(s)=1$ for $s \in \mathbb{Z}$. If $\varphi \in S^{p} A A(\mathbb{Z}, X)$, for any $\left\{\alpha_{n}^{\prime}\right\}$ in $\Pi$, there exist $\left\{\alpha_{n}\right\} \subset\left\{\alpha_{n}^{\prime}\right\}$ and $\tilde{\varphi} \in B S^{p}(\mathbb{T}, X)$ such that

$$
\left(\int_{[s, s+1]_{\mathbb{T}}}\left\|\varphi\left(r+\alpha_{n}\right)-\tilde{\varphi}(r)\right\|^{p} \Delta r\right)^{\frac{1}{p}} \rightarrow 0
$$

as $n \rightarrow \infty$ for each $s \in \mathbb{Z}$. Thus

$$
\int_{[s, s+1]_{\mathbb{T}}}\left\|\varphi\left(r+\alpha_{n}\right)-\tilde{\varphi}(r)\right\|^{p} \Delta r=\left\|\varphi\left(s+1+\alpha_{n}\right)-\tilde{\varphi}(s+1)\right\|^{p}+\left\|\varphi\left(s+\alpha_{n}\right)-\tilde{\varphi}(s)\right\|^{p} \rightarrow 0,
$$

as $n \rightarrow \infty$ for every $s \in \mathbb{Z}$. Then $\left\|\varphi\left(s+\alpha_{n}\right)-\tilde{\varphi}(s)\right\| \rightarrow 0$ as $n \rightarrow \infty$ for every $s \in \mathbb{Z}$. That is, $\varphi$ is an almost automorphic sequence on $\mathbb{Z}$.

From Definition 2.7, the space $S^{p} A A(\mathbb{T}, X)$ can inherit the following important properties from $A A\left(\mathbb{T}, B S^{p}(\mathbb{T}, X)\right)$ directly:

Proposition 2.1 Let $\varphi, \psi \in S^{p} A A(\mathbb{T}, X), l \in \Pi$, then

(i) $\varphi+\psi \in S^{p} A A(\mathbb{T}, X)$.

(ii) $\varphi(\cdot+l) \in S^{p} A A(\mathbb{T}, X)$.

(iii) $S^{p} A A(\mathbb{T}, X)$ is a Banach space under the norm $\|\cdot\|_{S^{p}}$.

Now we give the composition theorem on $\mathbb{T}$. Let

$$
\mathcal{T}(\varphi)(s):=\left(\frac{1}{\mathcal{L}} \int_{[s, s+\mathcal{L}]_{\mathbb{T}}}\|\varphi(r)\|^{p} \Delta r\right)^{\frac{1}{p}}, \quad \varphi \in B S^{p}(\mathbb{T}, X), s \in \mathbb{T} .
$$

In the proof, we will apply the following assumption:

(H) The operator $\mathcal{T}(\varphi(v(\cdot)))(s)$ is continuous on the set of $S^{p}$-bounded functions $v: \mathbb{T} \rightarrow \mathbb{R}$ at each $s \in \mathbb{T}$, that is, for any positive constant $\varepsilon$, we can find a positive constant $\delta$ such that if $\mathcal{T}\left(v_{1}-v_{2}\right)(s)<\delta$, then we have $\mathcal{T}\left(\varphi\left(v_{1}(\cdot)-v_{2}(\cdot)\right)\right)(s)<\varepsilon$ for each $s \in \mathbb{T}$. 
Theorem 2.2 Assume $\varphi \in B S^{p}(\mathbb{R}, \mathbb{R})$, and the above assumption $(\mathrm{H})$ is satisfied. If $v \in$ $S^{p} A A(\mathbb{T}, \mathbb{R}), \varphi(v(s-\gamma))$ is Stepanov-like almost automorphic on $\mathbb{T}$, where $\gamma \in \Pi$.

Proof Since $v \in S^{p} A A(\mathbb{T}, \mathbb{R})$, we know that for each $\left\{\alpha_{n}^{\prime}\right\} \subset \Pi$, there exist $\left\{\alpha_{n}\right\} \subset\left\{\alpha_{n}^{\prime}\right\}$ and $\tilde{v} \in B S^{p}(\mathbb{T}, \mathbb{R})$, satisfying

$$
\begin{aligned}
& \lim _{n \rightarrow \infty}\left(\frac{1}{\mathcal{L}} \int_{[s, s+\mathcal{L}]_{\mathbb{T}}}\left|v\left(r+\alpha_{n}\right)-\tilde{v}(r)\right|^{p} \Delta r\right)^{\frac{1}{p}}=0, \\
& \lim _{n \rightarrow \infty}\left(\frac{1}{\mathcal{L}} \int_{[s, s+\mathcal{L}]_{\mathbb{T}}}\left|\tilde{v}\left(r-\alpha_{n}\right)-v(r)\right|^{p} \Delta r\right)^{\frac{1}{p}}=0
\end{aligned}
$$

for every $s \in \mathbb{T}$. From the assumption $(\mathrm{H})$ and (2.2), we have

$$
\lim _{n \rightarrow \infty}\left(\frac{1}{\mathcal{L}} \int_{[s, s+\mathcal{L}]_{\mathbb{T}}}\left|\varphi\left(v\left(r+\alpha_{n}-\gamma\right)\right)-\varphi(\tilde{v}(r-\gamma))\right|^{p} \Delta r\right)^{\frac{1}{p}}=0
$$

pointwise on $s \in \mathbb{T}$. By a similar argument, we can get

$$
\lim _{n \rightarrow \infty}\left(\frac{1}{\mathcal{L}} \int_{[s, s+\mathcal{L}]_{\mathbb{T}}}\left|\varphi\left(\tilde{v}\left(r-\alpha_{n}-\gamma\right)\right)-\varphi(v(r-\gamma))\right|^{p} \Delta r\right)^{\frac{1}{p}}=0
$$

pointwise on $s \in \mathbb{T}$. Therefore, $\varphi(v(s-\gamma)) \in S^{p} A A(\mathbb{T}, \mathbb{R})$, and the proof is complete.

Remark 2.4 Theorem 3.19 in [11] showed a result about the composition of almost automorphic functions and continuous functions. Our result extends Theorem 3.19 to Stepanov-like almost automorphic functions by replacing "continuous" with respect to the sup norm by "continuous" with respect to the $S^{p}$ norm.

\section{The almost automorphy for a dynamic system on $\mathbb{T}$}

In this section, the concept of an exponential function on time scales will be given first. We say that $k: \mathbb{T} \rightarrow \mathbb{R}$ is regressive if $1+\mu(s) k(s) \neq 0$, for $s \in \mathbb{T}^{k}$. Denote

$$
\mathcal{R}=\mathcal{R}(\mathbb{T})=\mathcal{R}(\mathbb{T} ; \mathbb{R})=\left\{k: k \in C_{r d}(\mathbb{T}, \mathbb{R}), \text { and it is regressive }\right\}
$$

and

$$
\mathcal{R}^{+}=\mathcal{R}^{+}(\mathbb{T} ; \mathbb{R})=\{k \in \mathcal{R}, 1+\mu(s) k(s)>0, s \in \mathbb{T}\}
$$

Suppose that $k, l \in \mathcal{R}$ and define $k \oplus l$ and $\ominus k$ as follows:

$$
(k \oplus l)(s):=k(s)+l(s)+\mu(s) k(s) l(s), \quad(\ominus k)(s):=\frac{-k(s)}{1+\mu(s) k(s)},
$$

for $s \in \mathbb{T}^{k}$. We can easily check that the set $\mathcal{R}$ is an Abelian group with operation $\oplus$.

Definition 3.1 ([2]) Consider $k \in \mathcal{R}$. Set

$$
e_{k}(s, \tau)=\exp \left(\int_{[\tau, s]_{\mathbb{T}}} \varsigma_{\mu(r)}(k(r)) \Delta r\right) \quad \text { for } s, \tau \in \mathbb{T},
$$


with $\varsigma_{h}: C_{h} \rightarrow Z_{h}$ given by

$$
\varsigma_{h}(u)= \begin{cases}\frac{1}{h} \log (1+h u) & \text { for } h \neq 0, \\ u & \text { for } h=0 .\end{cases}
$$

The function log means the principal logarithm.

Proposition 3.1 ([13]) Suppose $\omega>0, s, \tau \in \mathbb{T}$. Then

(i) $e_{\ominus \omega}(s+l, \tau+l)=e_{\ominus \omega}(s, \tau)$ whenever $l \in \Pi$.

(ii) If $s$ is a point in $\mathbb{T}, e_{\ominus \omega}\left(s, \tau_{1}\right) \leq e_{\ominus \omega}\left(s, \tau_{2}\right)$, with $\tau_{1} \leq \tau_{2}$ and $\tau_{1}, \tau_{2} \in(-\infty, s]_{\mathbb{T}}$.

(iii) $\sum_{m=1}^{\infty} e_{\ominus \omega}(s, \sigma(s)-(m-1) \mathcal{L})$ is uniformly convergent for $s \in \mathbb{T}$. Furthermore, for every $s \in \mathbb{T}$,

$$
\sum_{m=1}^{\infty} e_{\ominus \omega}(s, \sigma(s)-(m-1) \mathcal{L}) \leq \lambda_{\omega}
$$

where $\lambda_{\omega}=\frac{1}{1-\exp (-\omega)}$, if $\mathbb{T}=\mathbb{R}$, and $\lambda_{\omega}=2+\omega \mu_{0}+\frac{1}{\omega \mu_{0}}$, if $\mathbb{T} \neq \mathbb{R}$, with $\mu_{0}=\sup _{s \in \mathbb{T}} \mu(s)$.

Now we are in a position to consider the following system:

$$
u^{\Delta}(s)=\mathcal{A}(s) u(s)+\varphi(s), \quad s \in \mathbb{T},
$$

where $\mathcal{A} \in \mathcal{R}\left(\mathbb{T}, \mathbb{R}^{n \times n}\right), \varphi$ is a continuous function, and $\varphi \in S^{p} A A\left(\mathbb{T}, \mathbb{R}^{n}\right)$. Let $U(s)$ be the fundamental solution of

$$
u^{\Delta}(s)=\mathcal{A}(s) u(s), \quad s \in \mathbb{T} .
$$

We say that equation (3.2) admits an exponential dichotomy on time scale $\mathbb{T}$ [3], if there exist a projection $P$ and positive constants $\omega, K$ such that

$$
\begin{aligned}
& \left|U(s) P U^{-1}(\tau)\right| \leq K e_{\ominus \omega}(s, \tau) \quad \text { if } s \geq \tau, \\
& \left|U(s)(I-P) U^{-1}(\tau)\right| \leq K e_{\ominus \omega}(\tau, s) \quad \text { if } s \leq \tau .
\end{aligned}
$$

Theorem 3.1 Let $\mathcal{A} \in A A\left(\mathbb{T}, \mathbb{R}^{n \times n}\right)$. Then, for $s \geq \tau$, the following condition is satisfied:

$$
\left|e_{\mathcal{A}}(s, \tau)\right| \leq K e_{\ominus \omega}(s, \tau)
$$

with $K>0$ and $\omega>0$. Then equation (3.1) admits a unique solution $u(s) \in A A(\mathbb{T}, \mathbb{R})$ given by

$$
u(s)=\int_{(-\infty, s]_{\mathbb{T}}} e_{\mathcal{A}}(s, \sigma(r)) \varphi(r) \Delta r
$$

for $s \in \mathbb{T}$. 
Proof By a similar argument as in the proof of Lemma 4.7 in [13], it is not difficult to show that the function in (3.4) is the unique bounded solution for (3.1). Now we proceed in two steps to prove it is almost automorphic.

Step 1 . We show the property of $e_{\mathcal{A}}(s, \sigma(\tau))$. From the definition of an almost automorphic function on $\mathbb{T}$, we know that for any $\left\{\alpha_{n}^{\prime}\right\}$ in $\Pi$, we can find $\left\{\alpha_{n}\right\} \subset\left\{\alpha_{n}^{\prime}\right\}$, and functions $\tilde{\mathcal{A}}, \tilde{\varphi}$, satisfying

$$
\left|\mathcal{A}\left(s+\alpha_{n}\right)-\tilde{\mathcal{A}}(s)\right| \rightarrow 0, \quad\left|\tilde{\mathcal{A}}\left(s-\alpha_{n}\right)-\mathcal{A}(s)\right| \rightarrow 0
$$

piecewise on $\mathbb{T}$ as $n \rightarrow \infty$, and

$$
\begin{aligned}
& \left(\frac{1}{\mathcal{L}} \int_{[s, s+\mathcal{L}]_{\mathbb{T}}}\left|\varphi\left(r+\alpha_{n}\right)-\tilde{\varphi}(r)\right|^{p} \Delta r\right)^{\frac{1}{p}} \rightarrow 0, \\
& \left(\frac{1}{\mathcal{L}} \int_{[s, s+\mathcal{L}]_{\mathbb{T}}}\left|\tilde{\varphi}\left(r-\alpha_{n}\right)-\varphi(r)\right|^{p} \Delta r\right)^{\frac{1}{p}} \rightarrow 0,
\end{aligned}
$$

piecewise on $\mathbb{T}$ as $n \rightarrow \infty$. Set

$$
\begin{aligned}
& \tilde{u}(s):=\int_{(-\infty, s]_{\mathbb{T}}} e_{\tilde{\mathcal{A}}}(s, \sigma(r)) \tilde{\varphi}(r) \Delta r \\
& W(s, \sigma(\tau)):=e_{\mathcal{A}}\left(s+\alpha_{n}, \sigma\left(\tau+\alpha_{n}\right)\right)-e_{\tilde{\mathcal{A}}}(s, \sigma(\tau)), \quad s \in \mathbb{T}, 0<s-\tau<\mathcal{L} .
\end{aligned}
$$

It is easy to get that

$$
\begin{aligned}
\frac{\partial \Delta}{} & \frac{W}{\partial \Delta s}(s(\tau)) \\
= & \mathcal{A}\left(s+\alpha_{n}\right) e_{\mathcal{A}}\left(s+\alpha_{n}, \sigma(\tau)+\alpha_{n}\right)-\tilde{\mathcal{A}}(s) e_{\tilde{\mathcal{A}}}(s, \sigma(\tau)) \\
= & \left(\mathcal{A}\left(s+\alpha_{n}\right)-\tilde{\mathcal{A}}(s)\right) e_{\mathcal{A}}\left(s+\alpha_{n}, \sigma(\tau)+\alpha_{n}\right) \\
& +\tilde{\mathcal{A}}(s)\left(e_{\mathcal{A}}\left(s+\alpha_{n}, \sigma(\tau)+\alpha_{n}\right)-e_{\tilde{\mathcal{A}}}(s, \sigma(\tau))\right) \\
= & \left(\mathcal{A}\left(s+\alpha_{n}\right)-\tilde{\mathcal{A}}(s)\right) e_{\mathcal{A}}\left(s+\alpha_{n}, \sigma(\tau)+\alpha_{n}\right)+\tilde{\mathcal{A}}(s) W(s, \sigma(\tau)) .
\end{aligned}
$$

Thus from the variation of constants formula, we have

$$
W(s, \sigma(\tau))=\int_{[\sigma(\tau), s]_{\mathbb{T}}} e_{\tilde{\mathcal{A}}}(s, \sigma(r))\left(\mathcal{A}\left(r+\alpha_{n}\right)-\tilde{\mathcal{A}}(r)\right) e_{\mathcal{A}}\left(r+\alpha_{n}, \sigma(\tau)+\alpha_{n}\right) \Delta r
$$

since $W(\sigma(\tau), \sigma(\tau))=0$. From (3.3), it is not difficult to verify that $\left\|e_{\tilde{\mathcal{A}}}(s, \tau)\right\| \leq K e_{\ominus \omega}(s$, $\tau), s \geq \tau$. Then

$$
\begin{aligned}
|W(s, \sigma(\tau))| & \leq \int_{[\sigma(\tau), s] \mathbb{T}}\left|e_{\tilde{\mathcal{A}}}(s, \sigma(r))\right| \cdot\left|\mathcal{A}\left(r+\alpha_{n}\right)-\tilde{\mathcal{A}}(r)\right| \cdot\left|e_{\mathcal{A}}\left(r+\alpha_{n}, \sigma(\tau)+\alpha_{n}\right)\right| \Delta r \\
& \leq \int_{[\sigma(\tau), s]_{\mathbb{T}}} K^{2}\left|\mathcal{A}\left(r+\alpha_{n}\right)-\tilde{\mathcal{A}}(r)\right| \cdot\left|e_{\ominus \omega}(s, \sigma(r)) e_{\ominus \omega}\left(r+\alpha_{n}, \sigma(\tau)+\alpha_{n}\right)\right| \Delta r \\
& =K^{2} e_{\ominus \omega}(s, \sigma(\tau)) \int_{[\sigma(\tau), s]]_{\mathbb{T}}}\left|\mathcal{A}\left(r+\alpha_{n}\right)-\tilde{\mathcal{A}}(r)\right| \cdot\left|e_{\ominus \omega}(r, \sigma(r))\right| \Delta r
\end{aligned}
$$




$$
=K^{2} e_{\ominus \omega}(s, \sigma(\tau)) \int_{[\sigma(\tau), s]_{\mathbb{T}}}\left|\mathcal{A}\left(r+\alpha_{n}\right)-\tilde{\mathcal{A}}(r)\right| \cdot|1+\omega \mu(r)| \Delta r .
$$

By (3.5) and [10, Theorem 2.1], for every $\varepsilon>0$, we can find $N_{1} \in \mathbb{Z}^{+}$such that, when $n>N_{1}$, we get

$$
\int_{[\sigma(\tau), s]_{\mathbb{T}}}\left|\mathcal{A}\left(r+\alpha_{n}\right)-\tilde{\mathcal{A}}(r)\right| \cdot|1+\omega \mu(r)| \Delta r<\varepsilon
$$

since on the interval $[\sigma(\tau), s]_{\mathbb{T}}$ the functions $\mathcal{A}(r), \tilde{\mathcal{A}}(r)$, and $1+\omega \mu(r)$ are all bounded. Thus if $0<s-\tau<\mathcal{L}$,

$$
\left|e_{\mathcal{A}}\left(s+\alpha_{n}, \sigma\left(\tau+\alpha_{n}\right)\right)-e_{\tilde{\mathcal{A}}}(s, \sigma(\tau))\right| \leq K^{2} \varepsilon e_{\ominus \omega}(s, \sigma(\tau))
$$

for $n>N_{1}, s \in \mathbb{T}$.

Step 2. We prove that $u$ is almost automorphic. Set

$$
\begin{aligned}
& K_{1}=\sum_{k=1}^{\infty} \int_{[s-k \mathcal{L}, s-(k-1) \mathcal{L}]_{\mathbb{T}}} e_{\mathcal{A}}\left(s+\alpha_{n}, \sigma\left(r+\alpha_{n}\right)\right)\left|\varphi\left(r+\alpha_{n}\right)-\tilde{\varphi}(r)\right| \Delta r, \\
& K_{2}=\sum_{k=1}^{\infty} \int_{[s-k \mathcal{L}, s-(k-1) \mathcal{L}]_{\mathbb{T}}}\left|e_{\mathcal{A}}\left(s+\alpha_{n}, \sigma\left(r+\alpha_{n}\right)\right)-e_{\tilde{\mathcal{A}}}(s, \sigma(r))\right| \cdot|\tilde{\varphi}(r)| \Delta r .
\end{aligned}
$$

By (3.6), for the $\varepsilon$ given above, there is $N \in \mathbb{Z}^{+}$such that $N>N_{1}$, and, when $n>N$, we have

$$
\left(\frac{1}{\mathcal{L}} \int_{[s, s+\mathcal{L}]_{\mathbb{T}}}\left|\varphi\left(r+\alpha_{n}\right)-\tilde{\varphi}(r)\right|^{p} \Delta r\right)^{\frac{1}{p}}<\varepsilon
$$

for every $s \in \mathbb{T}$. By Hölder inequality, (3.3), and Proposition 3.1, for $n>N$, $s \in \mathbb{T}$, we have

$$
\begin{aligned}
K_{1} \leq & \sum_{k=1}^{\infty}\left(\int_{[s-k \mathcal{L}, s-(k-1) \mathcal{L}]_{\mathbb{T}}}\left|e_{\mathcal{A}}\left(s+\alpha_{n}, \sigma\left(r+\alpha_{n}\right)\right)\right|^{q} \Delta r\right)^{\frac{1}{q}} \\
& \times\left(\int_{[s-k \mathcal{L}, s-(k-1) \mathcal{L}]_{\mathbb{T}}}\left|\varphi\left(r+\alpha_{n}\right)-\tilde{\varphi}(r)\right|^{p} \Delta r\right)^{\frac{1}{p}} \\
\leq & \sum_{k=1}^{\infty} K\left(\int_{[s-k \mathcal{L}, s-(k-1) \mathcal{L}]_{\mathbb{T}}}\left|e_{\ominus \omega}\left(s+\alpha_{n}, \sigma\left(r+\alpha_{n}\right)\right)\right|^{q} \Delta r\right)^{\frac{1}{q}} \\
& \times \mathcal{L}^{\frac{1}{p}}\left(\frac{1}{\mathcal{L}} \int_{[s-k \mathcal{L}, s-(k-1) \mathcal{L}]_{\mathbb{T}}}\left|\varphi\left(r+\alpha_{n}\right)-\tilde{\varphi}(r)\right|^{p} \Delta r\right)^{\frac{1}{p}} \\
= & \sum_{k=1}^{\infty} K\left(\int_{[s-k \mathcal{L}, s-(k-1) \mathcal{L}]_{\mathbb{T}}}\left|e_{\ominus \omega}(s, \sigma(r))\right|^{q} \Delta r\right)^{\frac{1}{q}} \\
& \times \mathcal{L}^{\frac{1}{p}}\left(\frac{1}{\mathcal{L}} \int_{[s-k \mathcal{L}, s-(k-1) \mathcal{L}]_{\mathbb{T}}}\left|\varphi\left(r+\alpha_{n}\right)-\tilde{\varphi}(r)\right|^{p} \Delta r\right)^{\frac{1}{p}} \\
\leq & \sum_{k=1}^{\infty} K \mid e_{\ominus \omega}\left(s, \sigma(s-(k-1) \mathcal{L}) \mid \cdot\left(\frac{1}{\mathcal{L}} \int_{[s-k \mathcal{L}, s-(k-1) \mathcal{L}]_{\mathbb{T}}}\left|\varphi\left(r+\alpha_{n}\right)-\tilde{\varphi}(r)\right|^{p} \Delta r\right)^{\frac{1}{p}}\right.
\end{aligned}
$$




$$
\leq K \lambda_{\omega} \varepsilon
$$

where $1 / p+1 / q=1$. By (3.7) and Proposition 3.1, we get for $n>N, s \in \mathbb{T}$,

$$
\begin{aligned}
K_{2} \leq & \sum_{k=1}^{\infty}\left(\int_{[s-k \mathcal{L}, s-(k-1) \mathcal{L}]_{\mathbb{T}}}|\tilde{\varphi}(r)|^{p} \Delta r\right)^{\frac{1}{p}} \\
& \times\left(\int_{[s-k \mathcal{L}, s-(k-1) \mathcal{L}]_{\mathbb{T}}}\left|e_{\mathcal{A}}\left(s+\alpha_{n}, \sigma\left(r+\alpha_{n}\right)\right)-e_{\tilde{\mathcal{A}}}(s, \sigma(r))\right|^{q} \Delta r\right)^{\frac{1}{q}} \\
\leq & \sum_{k=1}^{\infty}\left(\int_{[s-k \mathcal{L}, s-(k-1) \mathcal{L}]_{\mathbb{T}}}\left|K^{2} \varepsilon e_{\ominus \omega}(s, \sigma(r))\right|^{q} \Delta r\right)^{\frac{1}{q}} \mathcal{L}^{\frac{1}{p}}\|\tilde{\varphi}(r)\|_{S^{p}} \\
\leq & K^{2}\|\tilde{\varphi}(r)\|_{S^{p}} \varepsilon \mathcal{L}^{\frac{1}{p}} \sum_{k=1}^{\infty} e_{\ominus \omega}(s, \sigma(s)-(k-1) \mathcal{L}) \mathcal{L}^{\frac{1}{q}} \\
\leq & K^{2} \lambda_{\omega}\|\tilde{\varphi}(r)\|_{S^{p}} \varepsilon,
\end{aligned}
$$

where $1 / p+1 / q=1$. By (3.8) and (3.9), we know the series $K_{1}, K_{2}$ are convergent, and then we have

$$
\begin{aligned}
\left|u\left(s+\alpha_{n}\right)-\tilde{u}(s)\right| & =\left|\int_{\left(-\infty, s+\alpha_{n}\right]_{\mathbb{T}}} e_{\mathcal{A}}\left(s+\alpha_{n}, \sigma(r)\right) \varphi(r) \Delta r-\int_{(-\infty, s]_{\mathbb{T}}} e_{\tilde{\mathcal{A}}}(s, \sigma(r)) \tilde{\varphi}(r) \Delta r\right| \\
& \leq \int_{(-\infty, s]_{\mathbb{T}}}\left|e_{\mathcal{A}}\left(s+\alpha_{n}, \sigma\left(r+\alpha_{n}\right)\right) \varphi\left(r+\alpha_{n}\right)-e_{\tilde{\mathcal{A}}}(s, \sigma(r)) \tilde{\varphi}(r)\right| \Delta r \\
& =\sum_{k=1}^{\infty} \int_{[s-k \mathcal{L}, s-(k-1) \mathcal{L}]_{\mathbb{T}}}\left|e_{\mathcal{A}}\left(s+\alpha_{n}, \sigma\left(r+\alpha_{n}\right)\right) \varphi\left(r+\alpha_{n}\right)-e_{\tilde{\mathcal{A}}}(s, \sigma(r)) \tilde{\varphi}(r)\right| \Delta r \\
& \leq K_{1}+K_{2} \leq K \lambda_{\omega} \varepsilon+K \lambda_{\omega} \varepsilon .
\end{aligned}
$$

Thus for every point $s$ in $\mathbb{T}$, we get that $\lim _{n \rightarrow \infty}\left|u\left(s+\alpha_{n}\right)-\tilde{u}(s)\right|=0$. By a similar argument as in the above proof, we can show that $\lim _{n \rightarrow \infty}\left|\tilde{u}\left(s-\alpha_{n}\right)-u(s)\right|=0$ for every $s \in \mathbb{T}$. Therefore, the solution $u \in A A(\mathbb{T}, \mathbb{R})$, and this completes the proof.

Remark 3.1 (i) It is easy to verify that condition (3.3) is equivalent to the fact that the homogeneous equation $u^{\Delta}(s)=\mathcal{A}(s) u(s)$ admits an exponential dichotomy on $\mathbb{T}$.

(ii) Assume $\mathbb{T}=\mathbb{Z}$, then equation (3.1) is the difference equation $u(n+1)-u(n)=$ $\mathcal{A}(n) u(n)+\varphi(n)$. From Remark 2.3, we know that a Stepanov-like almost automorphic function on $\mathbb{Z}$ is equal to an almost automorphic sequence. Thus Theorem 3.1 is Theorem 3.8 in [15]. If $\mathbb{T}=\mathbb{R}$, it is clear that condition (3.3) can verify the exponential dichotomy condition on $\mathbb{R}$, and Theorem 3.1 can generalize Theorem 3.2 in [15]. That is, our Theorem 3.1 combines the two previous results, and generalizes them. 


\section{The almost automorphy for cellular neural networks on $\mathbb{T}$}

At this part, we will show the result about almost automorphy for the following system:

$$
\begin{aligned}
u_{l}^{\Delta}(s)= & -a_{l}(s) u_{l}(s)+\sum_{m=1}^{n} b_{l m}(s) F_{m}\left(u_{m}(s)\right) \\
& +\sum_{m=1}^{n} c_{l m}(s) F_{m}\left(u_{m}\left(s-\gamma_{l m}\right)\right)+I_{l}(s), \quad s \in \mathbb{T},
\end{aligned}
$$

for $l, m=1,2, \ldots, n, F_{m} \in C(\mathbb{R}, \mathbb{R}), a_{l}, b_{l m}, c_{l m} \in A A(\mathbb{T}, \mathbb{R}), \gamma_{l m} \in \Pi$, and where $I_{l}: \mathbb{T} \rightarrow \mathbb{R}$ are Stepanov-like almost automorphic on $\mathbb{T}$.

Let $a_{l}=\inf _{s \in \mathbb{T}} a_{l}(s)$. Now we give some assumptions:

$\left(\mathrm{H}_{1}\right)$ For each $m=1,2, \ldots, n$, there exist positive constants $C_{m}$ and $\alpha_{m}$ satisfying the following inequalities:

$$
\left|F_{m}(s)\right| \leq C_{m}, \quad\left|F_{m}(s)-F_{m}(t)\right| \leq \alpha_{m}|s-t|, \quad s, t \in \mathbb{R} .
$$

$\left(\mathrm{H}_{2}\right)$ For every $l=1,2, \ldots, n$, we have $\underline{a_{l}}>0,-a_{l}(s) \in \mathcal{R}^{+}$, with $s$ in $\mathbb{T}$.

$\left(\mathrm{H}_{3}\right) \lambda_{a_{l}}\left(\sum_{m=1}^{n} \alpha_{m}\left(\left\|b_{l m}\right\|+\left\|c_{l m}\right\|\right)\right)<1$, where $\lambda_{\underline{\underline{l}}}$ is given in Proposition 3.1.

By a similar argument as in the proof of [6, Lemma 2.15]], we can get the following result, and here we omit the detailed proof.

Lemma 4.1 Assume $\left(\mathrm{H}_{2}\right)$ is true, and $a_{l} \in A A(\mathbb{T}, \mathbb{R})$, for every $l=1,2, \ldots, n$. Then the homogeneous equation

$$
u^{\Delta}(s)=\mathcal{A}(s) u(s)
$$

satisfies the exponential dichotomy condition on $\mathbb{T}$, where

$$
\mathcal{A}(s)=\left(\begin{array}{llll}
-a_{1}(s) & & & \\
& -a_{2}(s) & & \\
& & \ddots & \\
& & & -a_{n}(s)
\end{array}\right) .
$$

Theorem 4.1 Let the assumptions $\left(\mathrm{H}_{1}\right)-\left(\mathrm{H}_{3}\right)$ hold, then (4.1) admits a unique almost automorphic solution $u^{*}(s)$.

Proof Let $E=\left\{\psi \in S^{p} A A\left(\mathbb{T}, \mathbb{R}^{n}\right):\|\psi\|_{S^{p}} \leq r_{0}\right\}$, where $r_{0}=\max _{1 \leq l \leq n}\left\{\lambda_{a_{l}}\left(\sum_{m=1}^{n} C_{m}\left(\left\|b_{l m}\right\|+\right.\right.\right.$ $\left.\left.\left.\left\|c_{l m}\right\|\right)+\left\|I_{l}\right\|_{S^{p}}\right)\right\}$. For any given $\psi=\left(\psi_{1}, \psi_{2}, \ldots, \psi_{n}\right)^{T} \in E$, we investigate the linear system:

$$
u_{l}^{\Delta}(s)=-a_{l}(s) u_{l}(s)+\sum_{m=1}^{n} b_{l m}(s) F_{m}\left(\psi_{m}(s)\right)+\sum_{m=1}^{n} c_{l m}(s) F_{m}\left(\psi_{m}\left(s-\gamma_{l m}\right)\right)+I_{l}(s)
$$

with $s \in \mathbb{T}$. By Lemma 4.1, the associated homogeneous equation

$$
u_{l}^{\Delta}(s)=-a_{l}(s) u_{l}(s)
$$


satisfies the exponential dichotomy condition with $l=1,2, \ldots, n$. By Proposition 2.1 and Theorem 2.2, we get that the function

$$
\Psi(s)=\left(\Psi_{1}(s), \Psi_{2}(s), \ldots, \Psi_{n}(s)\right)^{T}
$$

is Stepanov-like almost automorphic, where

$$
\Psi_{l}(s)=\sum_{m=1}^{n} b_{l m}(s) F_{m}\left(\psi_{m}(s)\right)+\sum_{m=1}^{n} c_{l m}(s) f_{m}\left(\psi_{m}\left(s-\gamma_{l m}\right)\right)+I_{l}(s),
$$

for $l=1,2, \ldots, n$. From Theorem 3.1, we get the conclusion that the equation $u^{\Delta}(s)=$ $\mathcal{A}(s) u(s)+\Psi(s)$ has a unique solution $u_{\psi}(s) \in A A\left(\mathbb{T}, \mathbb{R}^{n}\right)$, which is given by

$$
u_{\psi}(s)=\int_{(-\infty, s]_{\mathbb{T}}} e_{-\mathcal{A}}(s, \sigma(r)) \Psi(r) \Delta r=\left(u_{\psi_{1}}(s), u_{\psi_{2}}(s), \ldots, u_{\psi_{n}}(s)\right)^{T}, \quad s \in \mathbb{T},
$$

with $u(s)=\left(u_{1}(s), u_{2}(s), \ldots, u_{n}(s)\right)^{T}, \mathcal{A}(s)$ given in (4.2), and

$$
\begin{aligned}
u_{\psi_{l}}(s)= & \int_{(-\infty, s]_{\mathbb{T}}} e_{-a_{l}}(s, \sigma(r))\left(\sum_{m=1}^{n} b_{l m}(r) F_{m}\left(\psi_{m}(r)\right)\right. \\
& \left.+\sum_{m=1}^{n} c_{l m}(r) F_{m}\left(\psi_{m}\left(r-\gamma_{l m}\right)\right)+I_{l}(r)\right) \Delta r .
\end{aligned}
$$

Define the solution mapping

$$
\Gamma(\psi)(s)=\left(u_{\psi_{1}}(s), u_{\psi_{2}}(s), \ldots, u_{\psi_{n}}(s)\right)^{T}, \quad \psi \in E
$$

From Proposition 3.1, and $\left(\mathrm{H}_{1}\right)-\left(\mathrm{H}_{3}\right)$, we get

$$
\begin{aligned}
\left|u_{\psi_{l}}(s)\right|= & \mid \int_{(-\infty, s]_{\mathbb{T}}} e_{-a_{l}}(s, \sigma(r))\left(\sum_{m=1}^{n} b_{l m}(r) F_{m}\left(\psi_{m}(r)\right)\right. \\
& \left.+\sum_{m=1}^{n} c_{l m}(r) F_{m}\left(\psi_{m}\left(r-\gamma_{l m}\right)\right)+I_{l}(r)\right) \Delta r \mid \\
\leq & \left|\int_{(-\infty, s]_{\mathbb{T}}} e_{-\underline{a_{l}}}(s, \sigma(r))\left(\sum_{m=1}^{n}\left(b_{l m}(r)+c_{l m}(r)\right) C_{m}+I_{l}(r)\right) \Delta r\right| \\
= & \left|\sum_{k=1}^{\infty} \int_{[s-k \mathcal{L}, s-(k-1) \mathcal{L}]_{\mathbb{T}}} e_{-a_{l}}(s, \sigma(r))\left(\sum_{m=1}^{n}\left(b_{l m}(r)+c_{l m}(r)\right) C_{m}+I_{l}(r)\right) \Delta r\right| \\
\leq & \sum_{k=1}^{\infty}\left(\int_{[s-k \mathcal{L}, s-(k-1) \mathcal{L}]_{\mathbb{T}}}\left|e_{-\underline{a_{l}}}(s, \sigma(r))\right|^{q} \Delta r\right)^{\frac{1}{q}} \\
& \times \mathcal{L}^{\frac{1}{p}}\left(\frac{1}{\mathcal{L}} \int_{[s-k \mathcal{L}, s-(k-1) \mathcal{L}]_{\mathbb{T}}}\left|\sum_{m=1}^{n}\left(b_{l m}(r)+c_{l m}(r)\right) C_{m}+I_{l}(r)\right|^{p} \Delta r\right)^{\frac{1}{p}} \\
\leq & \sum_{k=1}^{\infty} e_{-\underline{a_{l}}}(s, \sigma(s-(k-1) \mathcal{L}))\left(\sum_{m=1}^{n} C_{m}\left(\left\|b_{l m}\right\|+\left\|c_{l m}\right\|\right)+\left\|I_{l}\right\|_{S^{p}}\right)
\end{aligned}
$$




$$
\leq \lambda_{\underline{a_{l}}}\left(\sum_{m=1}^{n} C_{m}\left(\left\|b_{l m}\right\|+\left\|c_{l m}\right\|\right)+\left\|I_{l}\right\|_{S^{p}}\right)=r_{0},
$$

for every $l=1,2, \ldots, n$. Thus $\|\Gamma(\psi)\|_{\infty} \leq r_{0}$, and $\Gamma(E) \subset E$.

Let $\psi, \chi \in E$. Then, by $\left(\mathrm{H}_{1}\right)$, we have

$$
\begin{aligned}
\left|u_{\psi_{l}}-u_{\chi_{l}}\right| & \mid \int_{\left(-\infty, s_{\mathbb{T}}\right.} e_{-a_{l}}(s, \sigma(r))\left(\sum_{m=1}^{n} b_{l m}(r)\left(F_{m}\left(\psi_{m}(r)\right)-F_{m}\left(\chi_{m}(r)\right)\right)\right. \\
& \left.+\sum_{m=1}^{n} c_{l m}(r)\left(F_{m}\left(\psi_{m}\left(r-\gamma_{l m}\right)\right)-F_{m}\left(\chi_{m}\left(r-\gamma_{l m}\right)\right)\right)\right) \Delta r \mid \\
\leq & \mid \int_{\left(-\infty, s_{\mathbb{T}}\right.} e_{-a_{l}}(s, \sigma(r))\left(\sum _ { m = 1 } ^ { n } \alpha _ { m } \left[b_{l m}(r)\left(\psi_{m}(r)-\chi_{m}(r)\right)\right.\right. \\
& \left.\left.+c_{l m}(r)\left(\psi_{m}\left(r-\gamma_{l m}\right)-\chi_{m}\left(r-\gamma_{l m}\right)\right)\right]\right) \Delta r \mid \\
\leq & \lambda_{a_{l}}\left(\sum_{m=1}^{n} \alpha_{m}\left(\left\|b_{l m}\right\|+\left\|c_{l m}\right\|\right)\right)\|\psi-\chi\|_{\infty} .
\end{aligned}
$$

By $\left(\mathrm{H}_{3}\right)$, we know that $\Gamma: E \rightarrow E$ is a contraction mapping. Thus we know that the operator $\Gamma$ has a unique fixed point $u^{*}(s)$ in $E$. Therefore, the system (4.1) admits a unique almost automorphic solution, and this completes the proof.

In the final, we give a specific example to explain the above Theorem 4.1 for the sake of clarity.

Example 4.1 Set $\mathbb{T}=\bigcup_{i=0}^{\infty}[2 i, 2 i+1]$. We can calculate easily that $\Pi=2 \mathbb{Z}, \mathcal{L}=2, \mu(s)=0$ for $s \in \Pi$. Consider the following neural network:

$$
\begin{aligned}
u_{l}^{\Delta}(s)= & -a_{l}(s) u_{l}(s)+\sum_{m=1}^{2} b_{l m}(s) F_{m}\left(u_{m}(s)\right) \\
& +\sum_{m=1}^{2} c_{l m}(s) F_{m}\left(u_{m}\left(s-\gamma_{l m}\right)\right)+I_{l}(s), \quad s \in \mathbb{T}, l=1,2,
\end{aligned}
$$

with

$$
\begin{aligned}
& a_{1}(s)=\sin \frac{\theta}{2+\cos 2 s+\cos \pi s}+2, \quad a_{2}(s)=\sin \frac{\theta}{2+\cos \pi s+\cos \sqrt{3} s}+2, \quad s \in \mathbb{T}, \\
& F_{1}(s)=\frac{1}{12} \cos ^{2} s, \quad F_{2}(s)=\frac{1}{12} \sin ^{2} s, \quad s \in \mathbb{R}, \\
& \left(b_{l m}(s)\right)_{2 \times 2}=\left(\begin{array}{cc}
\frac{1}{2}|\sin s|, & \frac{1}{2}|\cos s| \\
\frac{1}{3}|\sin s|, & \frac{1}{3}|\cos \sqrt{2} s|
\end{array}\right), \\
& \left(c_{l m}(s)\right)_{2 \times 2}=\left(\begin{array}{cc}
\frac{1}{5}|\cos s|, & \frac{1}{5}|\sin s| \\
\frac{1}{3}|\cos s|, & \frac{1}{3}|\sin \sqrt{2} s|
\end{array}\right), \quad s \in \mathbb{T},
\end{aligned}
$$




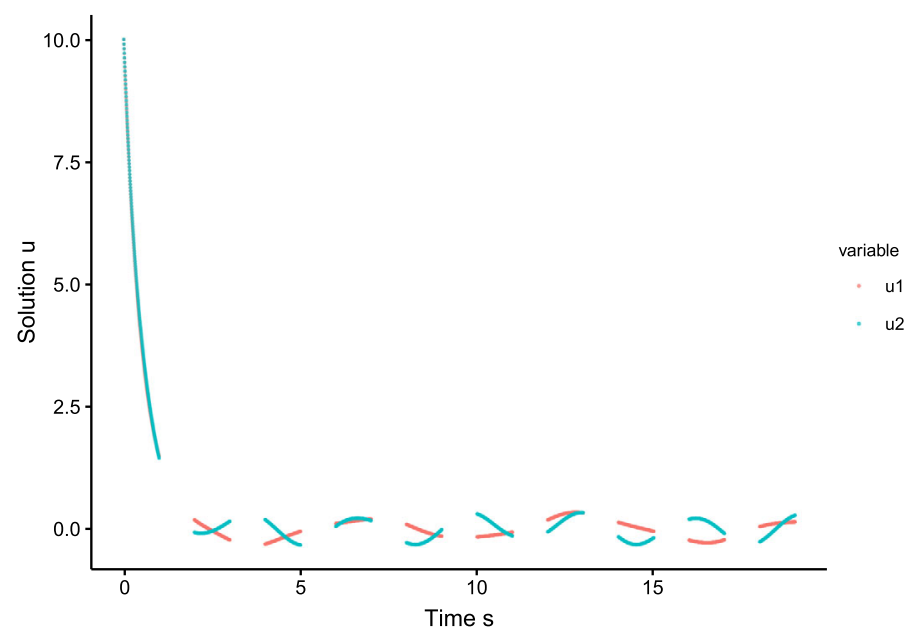

Figure 1 The unique almost automorphic solution $\left(u_{1}(s), u_{2}(s)\right)$ of $(4.4)$ on $\mathbb{T}$

$$
\begin{aligned}
& \gamma_{l m}=2 l m, \\
& I_{1}(s)=g_{1}(s) h_{1}(s), \quad I_{2}(s)=g_{2}(s) h_{2}(s),
\end{aligned}
$$

with

$$
\begin{aligned}
& g_{1}(s)=\left\{\begin{array}{ll}
1+s^{2}, & s \in 2 \mathbb{Z}, \\
\cos s, & s \in \mathbb{T} \backslash 2 \mathbb{Z},
\end{array} g_{2}(s)= \begin{cases}s, & s \in 2 \mathbb{Z}, \\
\cos 2 s, & s \in \mathbb{T} \backslash 2 \mathbb{Z},\end{cases} \right. \\
& h_{1}(s)=\left\{\begin{array}{ll}
1, & s \in 2 \mathbb{Z}, \\
\sin \frac{1}{2+\sin \sqrt{2} s}, & s \in \mathbb{T} \backslash 2 \mathbb{Z},
\end{array} \quad h_{2}(s)= \begin{cases}1, & s \in 2 \mathbb{Z}, \\
\cos \frac{1}{2+\cos \sqrt{2} s}, & s \in \mathbb{T} \backslash 2 \mathbb{Z},\end{cases} \right.
\end{aligned}
$$

where $\theta$ is a constant in $\mathbb{R}$, and $l, m=1,2$. It is clear that $a_{l} \in A A(\mathbb{T}, \mathbb{R})$ and $b_{l m}, c_{l m}, I_{l} \in$ $S^{p} A A(\mathbb{T}, \mathbb{R})$ with $l, m=1,2$. We can check easily that $F_{1}, F_{2}$ satisfy the condition $\left(\mathrm{H}_{1}\right)$ for $C_{1}=C_{2}=1 / 12, \alpha_{1}=\alpha_{2}=1 / 6$. We can calculate that $\underline{a_{1}}=1, \underline{a_{2}}=1$ easily, and then the condition $\left(\mathrm{H}_{2}\right)$ is true. It is clear that $\mu(2 i+1)=1$, and $\mu(s)=0, s \in[2 i, 2 i+1)$, for $i=0,1,2, \ldots$ Thus $\mu_{0}=1, \lambda_{\underline{a_{1}}}=2+\underline{a_{1}} \mu_{0}+\frac{1}{a_{1}}=4$, and $\lambda_{\underline{a_{2}}}=4$ similarly. By some easy calculation, we get $\left\|b_{1 m}\right\|=\frac{1}{2},\left\|b_{2 m}\right\|=\frac{1}{3},\left\|c_{1 m}\right\|=\frac{1}{5},\left\|c_{2 m}\right\|=\frac{1}{3}$, for $m=1,2$, and $\left\|I_{1}\right\|_{S^{p}}=1,\left\|I_{2}\right\|_{S^{p}}=1$. Thus we have

$$
\lambda_{\underline{a_{1}}} \sum_{m=1}^{2} \alpha_{m}\left(\left\|b_{1 m}\right\|+\left\|c_{1 m}\right\|\right)<1, \quad \lambda_{\underline{a_{2}}} \sum_{m=1}^{2} \alpha_{m}\left(\left\|b_{2 m}\right\|+\left\|c_{2 m}\right\|\right)<1,
$$

and $\left(\mathrm{H}_{3}\right)$ holds. From Theorem 4.1, we know that in the region $E=\left\{\psi \in S^{p} A A\left(\mathbb{T}, \mathbb{R}^{2}\right)\right.$ : $\left.\|\psi\|_{S^{p}} \leq r_{0}\right\}$, equation (4.1) admits a unique almost automorphic solution with $r_{0}=\frac{67}{60}$. The graph of the unique almost automorphic solution is shown as in Fig. 1. 
Funding

There was no funding support.

Availability of data and materials

No data, models, or code were generated or used during the study.

\section{Competing interests}

The authors declare that they have no competing interests.

Authors' contributions

LLZ proved the properties and the theorem, and wrote the paper. XDY reviewed and edited the manuscript. Both authors read and approved the final manuscript.

\section{Publisher's Note}

Springer Nature remains neutral with regard to jurisdictional claims in published maps and institutional affiliations.

Received: 3 November 2020 Accepted: 14 January 2021 Published online: 28 January 2021

\section{References}

1. Hilger, S.: Ein Maßkettenkalkül mit Anwendung auf Zentrumsmanningfaltigkeiten. PhD thesis, Universität Würzburg (1988)

2. Bohner, M., Peterson, A.: Dynamic Equations on Time Scales: An Introduction with Applications. Birkhäuser, Boston (2001)

3. Bohner, M., Peterson, A.: Dynamic Equations on Time Scales. Birkhäuser, Boston (2003)

4. Cabada, A., Vivero, D.R.: Expression of the Lebesgue $\Delta$-integral on time scales as a usual Lebesgue integral; application to the calculus of $\Delta$-antiderivatives. Math. Comput. Model. 43, 194-207 (2006)

5. Wang, Q.R., Zhu, Z.Q.: Almost periodic solutions of neutral functional dynamic systems in the sense of Stepanov, difference equations. In: Discrete Dynamical Systems and Applications, pp. 133-143 (2015)

6. Li, Y.K., Wang, C.: Almost periodic functions on time scales and applications. Discrete Dyn. Nat. Soc. (2011). https://doi.org/10.1155/2011/727068

7. Li, Y., Wang, C.: Uniformly almost periodic functions and almost periodic solutions to dynamic equations on time scales. Abstr. Appl. Anal. (2011). https://doi.org/10.1155/2011/341520

8. Lizama, C., Mesquita, J.G., Ponce, R.: A connection between almost periodic functions defined on timescales and $\mathbb{R}$. Appl. Anal. 12, 2547-2558 (2014)

9. Li, Y.K., Wang, C.: Pseudo almost periodic functions and pseudo almost periodic solutions to dynamic equations on time scales. Adv. Differ. Equ. (2012). https://doi.org/10.1186/1687-1847-2012-77

10. Li, Y.K., Zhao, L.L.: Weighted pseudo-almost periodic functions on time scales with applications to cellular neural networks with discrete delays. Math. Methods Appl. 40, 1905-1921 (2017)

11. Lizama, C., Mesquita, J.G.: Almost automorphic solutions of dynamic equations on time scales. J. Funct. Anal. 265 2267-2311 (2013)

12. Tang, C.H., Li, H.X.: Bochner-like transform and Stepanov almost periodicity on time scales with applications. Symmetry 10, 566 (2018). https://doi.org/10.3390/sym10110566

13. Tang, C.H., Li, H.X.: Stepanov-like pseudo almost periodic functions on time scales and applications to dynamic equations with delay. Open Math. 16, 826-841 (2018)

14. Dhama, S., Abbas, S.: Existence and stability of weighted pseudo almost automorphic solution of dynamic equation on time scales with weighted Stepanov-like $\left(S^{p}\right)$ pseudo almost automorphic coefficients. Qual. Theory Dyn. Syst. 19 46 (2020). https://doi.org/10.1007/s12346-020-00385-2

15. Zhang, L.L., Li, H.X.: Almost automorphic solutions for differential equations with piecewise constant argument. Bull. Aust. Math. Soc. 90, 99-112 (2014)

\section{Submit your manuscript to a SpringerOpen ${ }^{\circ}$ journal and benefit from:}

- Convenient online submission

- Rigorous peer review

- Open access: articles freely available online

- High visibility within the field

Retaining the copyright to your article

Submit your next manuscript at $>$ springeropen.com 